\title{
O paciente surdo e suas vivências no sistema de saúde: uma interface com a enfermagem
}

\author{
The deaf patient experiences in the health system: an interface \\ with the nursing
}

\author{
Vitor Machado Cavagna ${ }^{1} \bullet$ Wesley Pereira de Jesus Silva ${ }^{2}$ André Luiz de Souza Braga ${ }^{3}$ Marilda Andrade ${ }^{4}$
}

\begin{abstract}
RESUMO
Nos meios acadêmicos, a comunicação é um dos instrumentos básicos da enfermagem, porém, ao nos deparar com um paciente surdo, sem que tenhamos a capacitação necessária, o atendimento efetivo fica impossibilitado. Objetiva-se identificar como se dá o atendimento ao paciente surdo e analisar as barreiras de comunicação do profissional de saúde nestes atendimentos. A metodologia utilizada foi de estudo descritivo, de caráter exploratório, com abordagem qualitativa, no qual se realizou um levantamento bibliográfico sobre os aspectos gerais do processo de comunicação entre o profissional de saúde e o paciente surdo. Efetivou-se a coleta de dados através de um roteiro de entrevista semiestruturado, no Instituto Nacional de Educação de Surdos, localizado no bairro de Laranjeiras /RJ - Brasil. Os dados obtidos foram avaliados a partir do método de análise temática. Dos 15 participantes da pesquisa, 14 relataram situações de atendimento em saúde, nas quais, os profissionais não sabiam a Linguagem Brasileira de Sinais, retratando sentimento de exclusão neste paciente. Conclui-se a necessidade pela busca por capacitação dos profissionais de saúde no que tange à potencialização dos instrumentos utilizados nos processos de comunicação com esta demanda específica, oportunizando equidade e universalidade de acesso aos serviços de saúde.
\end{abstract}

Palavras-chave: Surdez; Barreiras de Comunicação; Linguagem de Sinais; Sistema de Saúde; Enfermagem.

\begin{abstract}
The communication is demonstrated in the academic times, as one of the basic instruments of the nursing, however, if we face a deaf patient and we do not have the necessary qualification the effective attendance stays disabled. Objective to identify how the treatment to a deaf patient is given in the health system and analyze the professional's communication barriers to the deaf patient. The used methodology was of descriptive study, with qualitative approach, in which a bibliographic survery happened on the general aspects of the communication process between the health professional and the deaf patient. It was accomplished, collects it of data through a semistructured script of interview, realized in the Instituto Nacional de Educação de Surdos (INES), nestled in Laranjeiras, RJ/Brazil. Of the 15 participants of the research, 14 had told situations of attendance in health, in which, the professionals did not know the Brazilian Language of Signals, depicting feeling of exclusion in this patient. It concludes that necessity for the search for health professionals' qualification in what it refers to the enhancement's instruments used in the processes of communication with this specific demand, providing equity and universality of health services access.
\end{abstract}

Keywords: Deafness; Communication Barriers; Signs Language; Health System; Nursing.

Não houve financiamento para a realização desta pesquisa. Texto aprovado no Comitê de Ética e Pesquisa do Hospital Universitário Antônio Pedro, da Universidade Federal Fluminense, no dia 03 de junho de 2011, e apresenta o n 121/11. CAAE: 0126.0.258.258-11, não havendo conflitos de interesse.

${ }^{1}$ Enfermeiro Especialista em Saúde Pública. Mestrando em Ciências do Cuidado em Saúde. Escola de Enfermagem Aurora de Afonso Costa/ UFF. E-mail:vcavagna@hotmail. com

${ }^{2}$ Mestre em Ciências do Cuidado em Saúde - Enfermeiro do Centro Municipal de Saúde Dr.Albert Sabin. Secretaria Municipal de Saúde do Rio de Janeiro. E-mail: wesley. jesus@uol.com.br

${ }^{3}$ Doutorando em Ciências do Cuidado em Saúde. Professor do Departamento de Fundamentos de Enfermagem e Administração. Escola de Enfermagem Aurora de Afonso Costa/UFF. E-mail: andre.braga@globo.com

${ }^{4}$ Doutora em Enfermagem. Vice-diretora e Professora da Escola de Enfermagem Aurora de Afonso Costa/ UFF. E-mail: marildaandrade@uol.com.br

Manuscrito baseado em trabalho de conclusão de curso de graduação em Enfermagem e Licenciatura, da Escola de Enfermagem Aurora de Afonso Costa. Niterói / RJ.

Autores: Vitor Machado Cavagna, Marilda Andrade (orientadora), Wesley Pereira de Jesus Silva (coorientador).

Título: 0 paciente surdo e suas vivências no sistema de saúde: uma interface com a enfermagem.

Trabalho apresentado em dezembro de 2011. 


\section{INTRODUÇÃO}

O desenvolvimento deste estudo deveu-se à reflexão sobre o atendimento à pessoa surda e sua inserção no sistema de saúde. Neste contexto, a comunicação se apresenta como um dos instrumentos básicos de trabalho do enfermeiro. Com isso, a contribuição quanto ao aprendizado de novos métodos de expressão com o paciente surdo é de extrema importância, pois se o profissional não consegue se comunicar com este cliente, inviabiliza-se o planejamento de cuidados para este usuário.

De acordo com pesquisas realizadas no ano de $2010^{1}$, cerca de 46 milhões de brasileiros possuíam algum tipo de deficiência (23,9\% da população), o que representa um percentual acima dos anos anteriores, quando se observou um contingente menor que $2 \%$. Visto que passou-se a atribuir o título de deficiente não só àqueles que se consideram incapazes, mas também àqueles que reportaram uma grande dificuldade permanente como caminhar, ouvir e enxergar ${ }^{2}$.

Nesse sentido, discorrer sobre deficiências depende de distintas percepções culturais e atitudes em relação às mesmas, como também na disponibilidade de serviços e legislação $0^{3}$, pois elas não são apenas resultado das condições saúde/doença, são também determinadas pelo contexto do meio ambiente físico e social.

A lei orgânica de saúde do Sistema Único de Saúde -SUS (Lei 8080/90) apresenta caráter de acessibilidade universal e gestão descentralizada, porém não engloba qualquer tipo de deficiência e irá tratar da ação governamental e das responsabilidades de cada setor a estes pacientes ${ }^{4}$. Determinando pleno acesso à saúde, à educação, a habilitação e reabilitação profissionais, ao trabalho, à cultura, ao desporto, ao turismo e ao lazer $^{5}$.

Diante da crise observada no setor público de saúde, não só a hospitalização, como também qualquer outro ambiente que exponha o binômio saúde/doença, passa por uma questão política e social, de modo que muitos profissionais de saúde reúnem-se para discutir a origem da doença e buscar soluções para tal problema. Contudo, não se preocupam em ressaltar a satisfação das necessidades básicas do ser humano ${ }^{6}$ como fatores indispensáveis para a promoção e preservação da saúde.

Através de comunicação estabelecida com o paciente, o profissional pode compreendê-lo como ser integral e também perceber sua visão de mundo. Para desenvolver um bom processo de trabalho, faz-se necessário que os enfermeiros usem a comunicação de maneira efetiva e, assim, evitem falhas na assistência. Não se pode pensar em atuação profissional sem levar em conta a importância do processo comunicativo e a união deste à prática assistencial ${ }^{7}$. Com isso, quando não há uma comunicação eficaz, não há como desenvolver a assistência pertinente a este paciente.

O processo comunicativo torna-se mais sério quando se apresenta com pacientes surdos, pois estes restringem sua comunicação a pessoas com as quais se relacionam habitualmente e, quando são hospitalizados ${ }^{8}$, passam a conviver em ambiente estranho, com pessoas que não os entendem.

Assim, o estudo objetivou identificar como o paciente surdo vivencia o sistema de saúde e o atendimento prestado no contexto do Sistema Único de Saúde (SUS), e analisar as barreiras da comunicação do profissional de saúde com o surdo.

\section{MÉTODO}

Trata-se de um estudo exploratório com abordagem qualitativa. 0 cenário da pesquisa foi o Instituto Nacional de Educação de Surdos (INES), localizado a Rua das Laranjeiras, $n^{\circ} 232$, no bairro das Laranjeiras, cidade do Rio de Janeiro - RJ - Brasil.

Os participantes da pesquisa foram 15 estudantes surdos do ensino médio e de ambos os sexos. Os critérios de inclusão foram: alunos maiores de 18 anos e que estiveram presentes no cenário de pesquisa durante a coleta de dados. Já os critérios de exclusão foram: aqueles que nunca passaram por situações de atendimento hospitalar e sem nenhuma patologia associada.

Desenvolveu-se um trabalho de campo, no qual houve a possibilidade de ter acesso ao local onde foi desenvolvida a pesquisa. A coleta de dados ocorreu por meio de observação participante e entrevista semiestruturada. A etapa de observação participante ocorreu durante as atividades desenvolvidas no local e, para a aplicação das entrevistas semiestruturadas, contou-se com a ajuda do intérprete no momento das entrevistas, sendo interlocutor entre o pesquisador e os participantes da pesquisa.

A importância e a legitimidade do trabalho do intérprete da Linguagem Brasileira de Sinais (LIBRAS) ainda encontrase em processo de construção, pois tal ofício ainda é muito recente $^{9}$. 0 intérprete tem o papel de orientar e interpretar a comunicação entre os surdos e ouvintes e de fazer a comunicação verbal e não verbal ${ }^{10}$.

Para a interpretação dos dados coletados, realizou-se a análise por categorias temáticas ${ }^{8}$, na busca dos núcleos do sentido que compõem um processo comunicativo, cuja presença ou frequência tenha algum significado para o objeto analítico visado.

Este trabalho obteve aprovação do Comitê de Ética e Pesquisa do Hospital Universitário Antônio Pedro, da Universidade Federal Fluminense, no dia 03 de junho de 2011, e apresenta o $n^{\circ}$ 121/11. CAAE: 0126.0.258.25811. Foram atribuídos nomes fictícios aos participantes da pesquisa.

\section{RESULTADOS}

\section{Aspectos gerais da população estudada}

Cada turma da instituição possui aproximadamente 15 alunos. Das turmas presentes no local no dia da pesquisa, 2 foram entrevistadas: $1^{\circ}$ e $3^{\circ}$ anos do ensino médio. 
abela 1. Turmas entrevistadas

\begin{tabular}{l|c|c|c}
\hline \multirow{2}{*}{ Entrevistados } & \multicolumn{3}{|c}{ Ensino Médio } \\
\cline { 2 - 4 } & $1^{\circ}$ ano & $2^{\circ}$ ano & $3^{\circ}$ ano \\
\hline Curso Noturno & 9 alunos & - & 4 alunos \\
\hline Curso Diurno & - & - & 2 alunos \\
\hline
\end{tabular}

Fonte: dados provenientes da pesquisa

A coleta de dados ocorreu após as 18 horas, devido à orientação da chefia de pesquisas da instituição e, neste horário, havia alunos de outros turnos sendo abordados sobre a participação no estudo, mostrando-se dispostos a contribuir com a pesquisa.

\section{PERFIL DOS PARTICIPANTES}

Foram realizados 2 dias de entrevistas com os participantes do estudo, totalizando 15 participantes, os quais são caracterizados na tabela abaixo.

A média da faixa etária encontrada foi 24,2 anos, sendo que $40 \%$ representam o sexo feminino e $60 \%$ masculino; a maioria dos entrevistados exerce atividades profissionais, o que demonstra que a limitação auditiva não tem se tornado entrave para inserção e permanência no mercado de trabalho, pois estão amparados por lei ${ }^{2}$ que estabelece a obrigatoriedade de empresas públicas e privadas de reservarem um quantitativo de vagas para esta parcela populacional.

O trabalho não constitui mera forma de sobrevivência e a sua importância ultrapassa as questões de ordem prática, contribuindo para a dignidade humana ${ }^{11}$. A estratégia adotada por diversas empresas consiste na tentativa de justificar o descumprimento da lei de cotas $^{4}$, sob o argumento de que suas atividades são caracterizadas por elevado grau de risco e periculosidade, o que tornaria não recomendável a contratação de pessoas com deficiência ${ }^{11}$.

A maioria dos entrevistados (80\%) se declarou solteira, possivelmente pela média da faixa etária que se encontravam e também por pesquisas que apontam que o matrimônio tem ocorrido no Brasil em idades mais avançadas. 0 padrão de idade de homens e mulheres disponíveis para o mercado matrimonial reflete as chances relativas de casamento e recasamento de cada um dos gêneros ${ }^{12}$. 0 relacionamento conjugal está associado à saúde e à qualidade de vida, principalmente nos anos de maturidade e velhice, porém o fato de um casamento durar não necessariamente significa que a união traga satisfação para os cônjuges ${ }^{13}$.

\section{DISCUSSÃO}

Como descrito anteriormente, a análise dos dados aconteceu a partir da confluência de sentido das respostas dadas após aplicação do roteiro de entrevistas, resultando nas seguintes categorias temáticas: (I) Comunicação em si - situação de atendimento em instituições de saúde; (II) Estratégias utilizadas para a comunicação com o profissional de saúde, (III) Conhecimento/desconhecimento por parte do profissional de saúde sobre a LIBRAS e (IV) Identificando a percepção do paciente em relação ao profissional. A seguir, apresenta-se a discussão dessas categorias.

Tabela 2. Características gerais dos participantes

\begin{tabular}{l|c|c|c|c}
\hline \multicolumn{1}{|c|}{ Nome } & Idade & Sexo & Profissão & Estado Civil \\
\hline Participante 1 & 20 & F & Rotulagem & Solteira \\
\hline Participante 2 & 27 & M & Desempregado & Solteiro \\
\hline Participante 3 & 22 & F & Desempregada & Solteira \\
\hline Participante 4 & 20 & M & Estudante & Solteiro \\
\hline Participante 5 & 25 & M & Estudante & Casado \\
\hline Participante 6 & 23 & M & Aux. Administrativo & Solteiro \\
\hline Participante 7 & 24 & M stoquista & Solteira \\
\hline Participante 8 & 35 & F & Aux. Operacional & Solteiro \\
\hline Participante 9 & 28 & M & Aux. de serv. gerais & Solteiro \\
\hline Participante 10 & 22 & M stoquista & Casada \\
\hline Participante 11 & 44 & F & Atendente & Solteiro \\
\hline Participante 12 & 22 & M & Aux. Administrativo & Solteira \\
\hline Participante 13 & 25 & F & Operadora de fábrica & Solteiro \\
\hline Participante 14 & 22 & Desempregado & Casada \\
\hline Participante 15 & 53 & D lar & \\
\hline
\end{tabular}

Fonte: dados provenientes da pesquisa 


\section{I - Comunicação em si - situação de atendimento em instituições de saúde.}

Quando os participantes foram questionados acerca da vivência nas instituições de saúde, emergiram as seguintes falas:

"Eu consegui me comunicar, porque domino a escrita [...] Geralmente quando tenho mais dificuldade, eu uso um intermediário, que é a minha mãe ou então uso intérprete." (Participante 4)

“Eu tinha que chamar intérprete, porque nesse caso, eu não tinha ninguém da família e se eu fosse sozinho, a doença não era realmente tratada, porque eles não entendiam o que eu sentia de fato, às vezes eu entendi o que ele tava me falando, mas ele não chegava ao meu objetivo." (Participante 5)

“Eu já cheguei num hospital, ninguém teve nenhum tipo de relacionamento comigo. A minha irmã foi junto comigo, aí ela sabe sinalizar e eu sou um pouco oralizada." (Participante 8)

“Eu passo, mostro o que tá acontecendo comigo, eu passo pro papel, aí o doutor consegue entender o que está acontecendo comigo." (Participante 9)

"Quando tem algum problema sério comigo, eu chamo intérprete; quando é uma coisa mais básica, eu não vou pro hospital." (Participante 10)

“Eu cheguei na unidade médica com meu marido [...] eu tinha que ter alguém pra me comunicar com o doutor, só que este não deixou que meu marido entrasse comigo, então eu peguei e fui embora."(Participante 11)

As dificuldades apresentadas nas respostas dos participantes quanto à vivência de utilização dos serviços de saúde nos fazem refletir acerca da necessidade de capacitação dos profissionais, visto que a grande maioria dos casos fez menção da presença de um acompanhante nas situações de atendimento. As barreiras de comunicação que se instauram entre o usuário dos serviços e o profissional justifica a necessidade de um acompanhante.

A saúde é um direito fundamental do ser humano, assegurado na lei. Sendo assim, cabe ao profissional de saúde se capacitar para promover a saúde da população, não excluindo este grupo específico (surdos), pois é dever do Estado garantir a saúde de toda a população ${ }^{14}$. Porém, a garantia de um atendimento inclusivo para este grupo deve resultar da capacitação profissional para um melhor atendimento, através do aprendizado da LIBRAS.

Há muitas barreiras no atendimento ao paciente surdo, entre as quais destacam-se as dificuldades linguísticas e a falta de confiança do paciente no profissional ouvinte, pois, muitas vezes, o surdo é comparado a deficientes mentais.

0 encontro com o paciente surdo pode ser esporádico, porém o desafio dos profissionais de saúde pode ir além dos serviços especializados e as barreiras acima apresentadas podem colocar em risco a assistência proporcionada ${ }^{15}$.
A utilização da escrita também se apresentou como um recurso facilitador para a interação profissional-paciente, mas é válido salientar que, em muitos dos casos, os participantes do estudo relataram não entender a grafia dos profissionais. Entretanto, o que foi mais observado é a dificuldade dos próprios participantes em relação a língua portuguesa, visto que a LIBRAS é sua língua materna, e a língua portuguesa é um aprendizado acessório.

O intérprete da LIBRAS também aparece como intermediário, porém este é requisitado quando não se tem a possibilidade de ter uma pessoa da família presente na situação de atendimento em saúde. É imprescindível lembrar que os participantes se sentem excluídos nos atendimentos, pois os profissionais não têm nenhum tipo de interação com eles, apenas se comunicam com o intermediário e acabam esquecendo que o paciente surdo é seu verdadeiro foco de atendimento.

Quando acontecem situações em que não se fazem presentes intérpretes ou qualquer tipo de intermediário, 0 atendimento ao paciente se dá de maneira inespecífica, visto que as dificuldades apresentadas pelo paciente são muitas, como a falta de compreensão da grafia e dificuldades com a língua portuguesa.

\section{II - Estratégias utilizadas para a comunicação com o profissional de saúde}

Dentre os recursos utilizados para a interação com o profissional de saúde, surgiram os seguintes depoimentos:

"A minha mãe sempre está comigo, com isso, ela serve de estratégia. Eu sempre utilizo minha mãe para me comunicar." (Participante 1)

"Todas as vezes que eu vou ao médico, sempre vou com o meu filho e o médico conversa com ele e o meu filho passa pra mim." (Participante 15)

"Gestos e mímicas são as estratégias utilizadas." (Participante 2)

"Escrita e leitura labial. Sempre gera um pouco de confusão, a comunicação não é perfeita". (Participante 3)

"Ele começou a escrever na folha, eu com o português tenho um pouco de dificuldade [...] Ele tava escrevendo uma coisa que eu não tava entendendo. A comunicação foi muito pouca comigo." (Participante 6)

"É difícil, porque eles falam, falam e escrevem no papel, só que a gente não conhece português direito." (Participante 10)

"Não conseguem se comunicar comigo, tentam falar, escrevem e eu não entendo nada. Ele é profissional, eu sei, mas a letra dele é muito difícil de ser entendida." (Participante 13)

"Ele escrevia, escrevia e eu não entendia nada. Eu pedia: Explica doutor, explica. Eu não entendi e fui pra casa, não adiantou eu ter ido lá." (Participante 14)

Com a finalidade de reduzir as dificuldades de comunicação, os participantes, em sua maioria, mencionam 
um intermediário como estratégia facilitadora neste processo. Contudo, não descartam a escrita, estratégia em que encontram dificuldade de entendimento, devido a dois fatores: a grafia dos profissionais e ainda, a própria língua portuguesa. A utilização de gestos, mímicas, leitura labial e a fala lenta também foram mencionados em alguns casos, porém estes são apresentados como meios que, em algumas vezes, podem não demonstrar o entendimento esperado.

A falta de iniciativa profissional para a interação com o paciente surdo é bem evidente, pois, na maioria dos casos apresentados, houve sempre uma estratégia por parte do paciente, o que nos faz refletir acerca da necessidade de maior envolvimento quanto à prestação do serviço de saúde e resolutividade dos problemas de saúde apresentados. Qualquer pessoa surda tem direito ao atendimento ${ }^{16}$, mas cabe aos profissionais ter as ferramentas mínimas para cuidar da pessoa portadora de um atendimento diferenciado e uma destas é se capacitar na linguagem de sinais.

$\mathrm{O}$ fato de a sociedade ainda conceber a existência de barreiras na comunicação pode ser explicado pelas dificuldades que a população tem em enxergar as pessoas com deficiência como pessoas produtivas e por acreditarem que elas são inferiores ${ }^{17}$. Por isso, atribuírem uma predisposição negativa e fazem um julgamento depreciativo a esta parcela da população.

\section{III - Conhecimento /desconhecimento por parte do profissional de saúde sobre a LIBRAS}

Em relação ao conhecimento e/ou desconhecimento da LIBRAS, obtiveram-se os seguintes resultados: dos 15 participantes, 14 relataram que os profissionais não sabiam/ desconheciam a linguagem de sinais. Apenas 1 relatou que o profissional sabia o alfabeto, porém não sinalizava.

A falta de capacitação profissional é demonstrada na maioria dos casos apresentados no estudo, e mesmo no único caso que o profissional sabia "soletrar" o alfabeto na linguagem de sinais, o atendimento não se deu de forma efetiva, pois não conseguiu atender as expectativas do participante que relatou o caso, como se observa na fala seguinte:

"... uma vez eu fui a um ginecologista e este sabia soletrar, não sabia sinalizar; só sabia o alfabeto e então eu desisti..." (Participante 11).

Existem várias formas de se comunicar, porém existe a dificuldade dos enfermeiros em comunicar-se com pacientes através de meios não verbais. Os profissionais precisam ser eficientes no desempenho do seu papel, proporcionando melhoria na relação com este tipo de paciente ${ }^{18,7}$.

\section{IV - Identificando a percepção do paciente em relação ao profissional}

No que diz respeito à percepção do paciente sobre o atendimento profissional, apresentamos as seguintes falas:
“Eu me sinto destratada." (Participante 1)

"Parece que não tem nada de concreto, que não é um atendimento, não tem nada que me atenda de verdade." (Participante 15)

"O atendimento não é digno para a pessoa que é surda." (Participante 2)

"Me sinto chateada, pois parece que o atendimento não é direcionado pra mim." (Participante 3)

"Eu sinto como se toda sociedade excluísse." (Participante 4)

"Eu me sinto discriminado, sinto que o atendimento que deve ser prestado a mim, não é prestado." (Participante 5)

"É muito difícil esse atendimento, é um assunto pesado, é muito difícil esse relacionamento." (Participante 6)

“Tem gente que não tem experiência com a gente, é uma forma diferenciada, né? É muito difícil pra gente que é surdo." (Participante 7)

"Os profissionais não dão muito valor, pois toda vez eu tenho que chamar minha irmã, e com isso eu fico muito incomodada." (Participante 8)

"Fico meio triste com isso, meio chateado com eles, por não ter essa comunicação [...] as pessoas têm que aprender a conviver com os surdos." (Participante 9)

"Eu fico com raiva disso, triste, chateado, com raiva. (Participante 10)

Eu fico chateada, pois o surdo sofre muito ." (Participante 11)

“Pra mim é normal, só que eu sei que existem pessoas que não sabem se comunicar comigo e é difícil, né? É muito importante que se tenha essa comunicação com a gente." (Participante 12)

“É um ar de discriminação. Com o ouvinte é fácil, se fala o que pode e o que não pode, como está a saúde, mas pra gente é difícil". (Participante 13)

"Fico chateado devido a falta de intérprete e de comunicação com os profissionais." (Participante 14)

É notória a opinião dos participantes no que diz respeito à falta de comunicação dos profissionais; muitos se sentem destratados, indignados, chateados e discriminados, já que o atendimento não supre suas necessidades. Com isso, muitos deles procuravam as instituições de saúde, apenas nos momentos de extrema necessidade e, na maioria dos casos, não tinham atendimento satisfatório.

Referindo-se à percepção do paciente acerca do atendimento nos serviços de saúde, há relatos que este expressa seus sentimentos como uma descrição particular de um momento vivenciado por ele ${ }^{19}$, considerando que sentimento é o símbolo utilizado para descrever a consciência de estar a "mercê" dos cuidados dos profissionais, porém, nem sempre, estes cuidados suprem suas necessidades.

A insatisfação presente na fala de todos os participantes nos faz refletir acerca da humanização no atendimento em saúde, visto que o despreparo profissional para a prestação de ações para este segmento populacional 
resulta no descrédito dos pacientes surdos em relação aos serviços de saúde, pois, como foi explicitado pelos próprios participantes deste estudo, a comunicação ainda não acontece de maneira efetiva, sempre existindo algum entrave neste processo.

\section{CONCLUSÃO}

A percepção do paciente surdo acerca do profissional de saúde ainda é feita pelo distanciamento. Não se tem a total confiança para um atendimento, pois a falta de comunicação existente neste binômio se apresenta de maneira complexa.

A humanização do cuidado deve ser idealizada dentro de perspectivas que incluam os que necessitam de atendimento especializado, conforme demonstrado neste estudo. As ações voltadas ainda se encontram deficitárias para esta parcela da população, evidenciando um despreparo profissional.

A oferta de ferramentas mínimas na aprendizagem da LIBRAS oportunizariam aos profissionais uma forma inclusiva de atenção aos pacientes surdos. Flexibilizar o horário de trabalho destes profissionais para que possam se qualificar em relação aos aspectos da comunicação a torna efetiva, atendendo as expectativas do paciente em relação aos profissionais. Cabe a estes refletir acerca das necessidades dos pacientes, evitando constrangimentos e oportunizando uma melhor interação.

A inserção da disciplina de LIBRAS no currículo acadêmico desvelou a relevância na construção de um profissional, através do processo comunicativo, com competências para reconhecer as necessidades desta parcela populacional em sua integralidade, e, assim, superar falhas nos serviços de saúde e credibilizar a relação profissional/paciente.

\section{REFERÊNCIAS}

1. Brasil. Ministério do Planejamento, Orçamento e Gestão. Instituto Brasileiro de Geografia e Estatística. Censo Demográfico 2010. Características gerais da população, religião e pessoas com deficiência. [Internet] Brasília. 2010 [acesso atualizado em 02 novembro 2016]; Disponível em: http://biblioteca.ibge.gov.br/bibliotecacatalogo?view=detalhes \&id=794

2. Brasil. Fundação Oswaldo Cruz. Instituto de Comunicação e Informação Científica e Tecnologia em Saúde. Política Nacional de Saúde da Pessoa Portadora de Deficiência. Rio de Janeiro, RJ. [Internet] Rio de Janeiro. 2010. [acesso atualizado em 02 novembro 2016]; Disponível em http://www.saudeidoso.icict.fiocruz.br/pdf/ politicanacionaldesaudepessoaportadoradedeficiencia.pdf

3. Farias N, Buchalla CM. A Classificação Internacional de Funcionalidade, Incapacidade e Saúde da Organização Mundial da Saúde: Conceitos, Usos e Perspectivas. Rev Bras de Epidemiol [Internet] 2005[acesso em 02 novembro 2016]; (8): 187-93. Disponível em http://www.scielo.br/pdf/rbepid/v8n2/11.pdf
4. Casa Civil. Subchefia de Assuntos Jurídicos - Decreto n³298. Política Nacional para Integração da Pessoa com Deficiência. [Internet] Brasília. 1999. [acesso atualizado em 02 novembro 2016]; Disponível em http://www.planalto.gov.br/ccivil_03/ decreto/d3298.htm

5. Bernardes LCG, Maior IML, Spezia CH, Araújo TCCF. Pessoas com deficiência e políticas de saúde no Brasil: reflexões bioéticas. Rev Ciência e Saúde Colet. [Internet] Brasília. 2009. [acesso atualizado em 02 novembro 2016]; v.14(1). Disponível em http://biblioteca.unipac.br/cgi-bin/ wxis.exe?IsisScript=phl8/003.xis \&cipar=valadares $/ p h l$. cip \&bool=exp\&opc=decorado \&exp=PESSOAS\%20COM $\% 20$ DEFICIENCIA\& code $=\&$ lang $=$

6. Almeida IS, Rodrigues BMRD, Simões SMF. Desvelando o cotidiano do adolescente hospitalizado. Rev Bras de Enfermag. [Internet] Rio de Janeiro. 2005. [acesso atualizado em 02 novembro 2016]; mar-abr; 58(2):147-51. Disponível em: http://www.scielo.br/pdf/reben/v58n2/a03.pdf

7. Pagliuca LMF, Fiúza NLG, Rebouças CBA. Aspectos da comunicação da enfermeira com o deficiente auditivo. Rev da Esc de Enfermag da USP. [Internet] São Paulo. 2007. [acesso atualizado em 02 novembro 2016]; 41(3):411-8 . Disponível em http://www.scielo.br/pdf/reeusp/v41n3/10.pdf

8. Garcia CL, Pereira HC, Marinho MNASB. Percepções da mulheres acerca do exame de prevenção câncer cérvicouterino. Rev Bras de Enfermag. [Internet] São Paulo. 2010. [acesso atualizado em 05 novembro 2016]; v2, n.10. Disponível em http://www.sumarios.org/sites/default/files/ pdfs/60759_6438.PDF

9. Souza SRG, Figueiredo AM. Como elaborar projetos, monografias, dissertações e teses. $1^{a}$ Ed. São Paulo. Lumen Juris 2010. p.30-5.

10. Araújo EABS, Ferraz FB. O conceito da pessoa com deficiência e seu impacto nas ações afirmativas brasileiras e no mercado de trabalho. In Anais do XIX Encontro Nacional do Compedi . Jun 09-12 [Internet] Fortaleza. 2010. [acesso atualizado em 05 novembro 2016]. Disponível em http://www.repositorio. ufc.br:8080/ri/bitstream/123456789/541/1/2010_eve_ fbferraz.pdf

11. Ribeiro MCMA, Carneiro R. A inclusão indesejada: as empresas brasileiras face a lei de cotas para pessoas com deficiência no mercado de trabalho. Rev OES. [Internet] Bahia. 2009. [acesso atualizado em 05 novembro 2016]; v 16 - n.50. Disponível em www.revistaoes.ufba.br

12. Oliveira MC. A família brasileira no limiar dos anos 2000. Rev Est Fem. [Internet] São Paulo. 2010. [acesso atualizado em 05 novembro 2016]; v 12. Disponível em http://www.periodicos. ufsc.br/index.php/ref/article/view/16657/15219

13. Comin FS, Santos MAS. Casar e ser feliz: mapeando a mensuração da satisfação conjugal. Rev Psico. [Internet] São Paulo.2009. [acesso atualizado em 05 novembro 2016]; v40, n.4. Disponível em http://revistaseletronicas.pucrs.br/ojs/ index.php/revistapsico/article/viewFile/4512/4928

14. Brasil. Casa Civil, Subchefia de Assuntos Jurídicos. Lei n8080/90. [Internet] Brasília.1990. [acesso atualizado em 05 novembro 2016]; Disponível em http://www.planalto.gov.br/ ccivil_03/leis/l8080.htm

15. Chaveiro N, Barbosa MA, Porto CC. Revisão de literatura sobre o atendimento ao paciente surdo pelos profissionais da saúde. 
Rev da Escol de Enfermag USP. [Internet] São Paulo. 2008 .[acesso atualizado em 02 novembro 2016]; v42(3): 57883 Disponível em http://www.scielo.br/pdf/reeusp/v42n3/ v42n3a22.pdf

16. Brasil. Câmara dos Deputados. Lei № 10.436/2002 - Legislação de Libras. [Internet] Brasília. 2002. [acesso atualizado em 02 novembro 2016]; Disponível em http://www.planalto.gov.br/ ccivil_03/leis/2002//10436.htm

17. Furrer, MA. Tipos de Barreira. In Acessibilidade na Prática. [Internet] São Paulo. 2012. [acesso atualizado em 02 novembro 2016]; Disponível em http://www.acessibilidadenapratica. com.br/textos/tipos-de-barreiras

18. Rosa CG, Barbosa MA, Bachion MM. Comunicação da equipe de enfermagem com o deficiente auditivo com surdez severa: um estudo exploratório. Rev Elet de Enfermag. [Internet] Goiânia. 2009. [acesso atualizado em 02 novembro 2016]. v2. Disponível em www.fen.ufg.br/revistas

19. Garcia CL, Pereira HC, Marinho MNASB. Percepções da mulheres acerca do exame de prevenção câncer cérvicouterino. Rev Brasilei em Promo da Saúde. RBPS. [Internet] Fortaleza. 2010 [acesso atualizado em 02 novembro 2016]; 23(2): 118-125, abr./jun . Disponível em http://ojs.unifor.br/ index.php/RBPS/article/view/2005/2301 